\title{
A Centrally Symmetric Version of the Cyclic Polytope
}

\author{
Alexander Barvinok • Isabella Novik
}

Received: 28 December 2006 / Revised: 2 August 2007 /

Published online: 18 September 2007

(C) Springer Science+Business Media, LLC 2007

\begin{abstract}
We define a centrally symmetric analogue of the cyclic polytope and study its facial structure. We conjecture that our polytopes provide asymptotically the largest number of faces in all dimensions among all centrally symmetric polytopes with $n$ vertices of a given even dimension $d=2 k$ when $d$ is fixed and $n$ grows. For a fixed even dimension $d=2 k$ and an integer $1 \leq j<k$ we prove that the maximum possible number of $j$-dimensional faces of a centrally symmetric $d$-dimensional polytope with $n$ vertices is at least $\left(c_{j}(d)+o(1)\right)\left(\begin{array}{c}n \\ j+1\end{array}\right)$ for some $c_{j}(d)>0$ and at most $\left(1-2^{-d}+o(1)\right)\left(\begin{array}{c}n \\ j+1\end{array}\right)$ as $n$ grows. We show that $c_{1}(d) \geq 1-(d-1)^{-1}$ and conjecture that the bound is best possible.
\end{abstract}

\section{Introduction and Main Results}

To characterize the numbers that arise as the face numbers of simplicial complexes of various types is a problem that has intrigued many researchers over the last half century and has been solved for quite a few classes of complexes, among them the class of all simplicial complexes $[13,14]$ as well as the class of all simplicial polytopes $[4,22]$. One of the precursors of the latter result was the Upper Bound Theorem (UBT, for short) [16] that provided sharp upper bounds on the face numbers of all

Research of A. Barvinok partially supported by NSF grant DMS 0400617.

Research of I. Novik partially supported by Alfred P. Sloan Research Fellowship and NSF grant DMS-0500748.

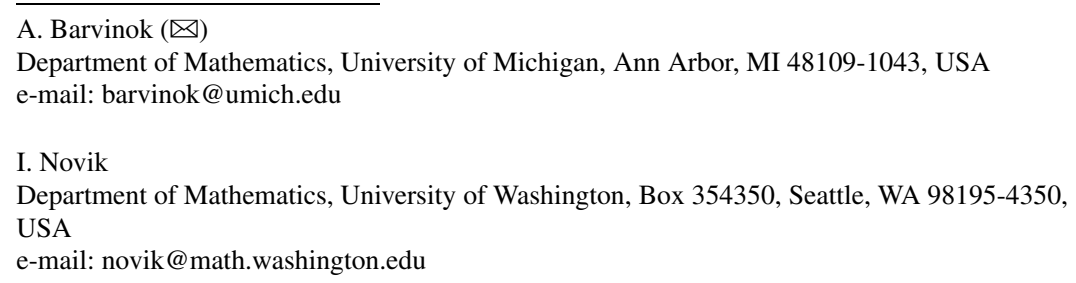


$d$-dimensional polytopes with $n$ vertices. While the UBT is a classic by now, the situation for centrally symmetric polytopes is wide open. For instance, the largest number of edges, $\operatorname{fmax}(d, n ; 1)$, that a $d$-dimensional centrally symmetric polytope on $n$ vertices can have is unknown even for $d=4$. Furthermore, no plausible conjecture about the value of $\operatorname{fmax}(d, n ; 1)$ exists.

In this paper, we establish certain bounds on $\operatorname{fmax}(d, n ; 1)$ and, more generally, on $\operatorname{fmax}(d, n ; j)$, the maximum number of $j$-dimensional faces of a centrally symmetric $d$-dimensional polytope with $n$ vertices. For every even dimension $d$ we construct a centrally symmetric polytope with $n$ vertices, which, we conjecture, provides asymptotically the largest number of faces in every dimension as $n$ grows and $d$ is fixed among all $d$-dimensional centrally symmetric polytopes with $n$ vertices, see the discussion after Theorem 1.4 for the precise statement of the conjecture.

Let us recall the basic definitions. A polytope will always mean a convex polytope (that is, the convex hull of finitely many points), and a $d$-polytope-a $d$-dimensional polytope. A polytope $P \subset \mathbb{R}^{d}$ is centrally symmetric (cs, for short) if for every $x \in P$, $-x$ belongs to $P$ as well, that is, $P=-P$. The number of $i$-dimensional faces ( $i$-faces, for short) of $P$ is denoted $f_{i}=f_{i}(P)$ and is called the $i$ th face number of $P$.

The UBT proposed by Motzkin in 1957 [17] and proved by McMullen [16] asserts that among all $d$-polytopes with $n$ vertices, the cyclic polytope, $C_{d}(n)$, maximizes the number of $i$-faces for every $i$. Here the cyclic polytope, $C_{d}(n)$, is the convex hull of $n$ distinct points on the moment curve $\left(t, t^{2}, \ldots, t^{d}\right) \in \mathbb{R}^{d}$ or on the trigonometric moment curve $(\cos t, \sin t, \cos 2 t, \sin 2 t, \ldots, \cos k t, \sin k t) \in \mathbb{R}^{2 k}(\operatorname{assuming} d=2 k)$. Both types of cyclic polytopes were investigated by Carathéodory [5] and later by Gale [10] who, in particular, showed that the two types are combinatorially equivalent (for even $d$ ) and independent of the choice of points. Cyclic polytopes were also rediscovered by Motzkin [12, 17] and many others. We refer the readers to [2] and [23] for more information on these amazing polytopes.

Here we define and study a natural centrally symmetric analogue of cyclic polytopes-bicyclic polytopes.

\subsection{The Symmetric Moment Curve and Bicyclic Polytopes}

Consider the curve

$$
\mathrm{SM}_{2 k}(t)=(\cos t, \sin t, \cos 3 t, \sin 3 t, \ldots, \cos (2 k-1) t, \sin (2 k-1) t) \quad \text { for } t \in \mathbb{R},
$$

which we call the symmetric moment curve, $\operatorname{SM}_{2 k}(t) \in \mathbb{R}^{2 k}$. The difference between $\mathrm{SM}_{2 k}$ and the trigonometric moment curve is that we employ only odd multiples of $t$ in the former. Clearly, $\mathrm{SM}_{2 k}(t+2 \pi)=\mathrm{SM}_{2 k}(t)$, so $\mathrm{SM}_{2 k}$ defines a map $\mathrm{SM}_{2 k}$ : $\mathbb{R} / 2 \pi \mathbb{Z} \rightarrow \mathbb{R}^{2 k}$. It is convenient to identify the quotient $\mathbb{R} / 2 \pi \mathbb{Z}$ with the unit circle $\mathbb{S}^{1} \subset \mathbb{R}^{2}$ via the map $t \mapsto(\cos t, \sin t)$. In particular, $\{t, t+\pi\}$ is a pair of antipodal points in $\mathbb{S}^{1}$. We observe that

$$
\operatorname{SM}_{2 k}(t+\pi)=-\mathrm{SM}_{2 k}(t)
$$

so the symmetric moment curve $\mathrm{SM}_{2 k}\left(\mathbb{S}^{1}\right)$ is centrally symmetric about the origin. 
Let $X \subset \mathbb{S}^{1}$ be a finite set. A bicyclic $2 k$-dimensional polytope, $\mathcal{B}_{2 k}(X)$, is the convex hull of the points $\mathrm{SM}_{2 k}(x), x \in X$ :

$$
\mathcal{B}_{2 k}(X)=\operatorname{conv}\left(\operatorname{SM}_{2 k}(X)\right) \text {. }
$$

We note that $\mathcal{B}_{2 k}(X)$ is a centrally symmetric polytope as long as one chooses $X$ to be a centrally symmetric subset of $\mathbb{S}^{1}$. In the case of $k=2$ these polytopes were introduced and studied (among certain more general 4-dimensional polytopes) by Smilansky [20, 21], but to the best of our knowledge the higher-dimensional bicyclic polytopes have not yet been investigated. Also, in [20] Smilansky studied the convex hull of $\mathrm{SM}_{4}\left(\mathbb{S}^{1}\right)$ (among convex hulls of certain more general 4-dimensional curves) but the convex hull of higher dimensional symmetric moment curves has not been studied either.

We recall that a face of a convex body is the intersection of the body with a supporting hyperplane. Faces of dimension 0 are called vertices and faces of dimension 1 are called edges. Our first main result concerns the edges of the convex hull

$$
\mathcal{B}_{2 k}=\operatorname{conv}\left(\operatorname{SM}_{2 k}\left(\mathbb{S}^{1}\right)\right)
$$

of the symmetric moment curve. Note that $\mathcal{B}_{2 k}$ is centrally symmetric about the origin.

Let $\alpha \neq \beta \in \mathbb{S}^{1}$ be a pair of non-antipodal points. By the arc with the endpoints $\alpha$ and $\beta$ we always mean the shorter of the two arcs defined by $\alpha$ and $\beta$.

Theorem 1.1 For every positive integer $k$ there exists a number

$$
\frac{2 k-2}{2 k-1} \pi \leq \psi_{k}<\pi
$$

with the following property: if the length of the arc with the endpoints $\alpha \neq \beta \in \mathbb{S}^{1}$ is less than $\psi_{k}$, then the interval $\left[\operatorname{SM}_{2 k}(\alpha), \operatorname{SM}_{2 k}(\beta)\right]$ is an edge of $\mathcal{B}_{2 k}$; and if the length of the arc with the endpoints $\alpha \neq \beta \in \mathbb{S}^{1}$ is greater than $\psi_{k}$, then the interval $\left[\mathrm{SM}_{2 k}(\alpha), \mathrm{SM}_{2 k}(\beta)\right]$ is not an edge of $\mathcal{B}_{2 k}$.

It looks quite plausible that

$$
\psi_{k}=\frac{2 k-2}{2 k-1} \pi
$$

and, indeed, this is the case for $k=2$, cf. Sect. 4 .

One remarkable property of the convex hull of the trigonometric moment curve in $\mathbb{R}^{2 k}$ is that it is $k$-neighborly, that is, the convex hull of any set of $k$ distinct points on the curve is a $(k-1)$-dimensional face of the convex hull. The convex hull of the symmetric moment curve turns out to be locally $k$-neighborly.

Theorem 1.2 For every positive integer $k$ there exists a number $\phi_{k}>0$ such that if $t_{1}, \ldots, t_{k} \in \mathbb{S}^{1}$ are distinct points that lie on an arc of length at most $\phi_{k}$, then

$$
\operatorname{conv}\left(\mathrm{SM}_{2 k}\left(t_{1}\right), \ldots, \mathrm{SM}_{2 k}\left(t_{k}\right)\right)
$$

is a $(k-1)$-dimensional face of $\mathcal{B}_{2 k}$. 
From Theorems 1.1 and 1.2 on one hand and using a volume trick similar to that used in [15] on the other hand, we prove the following results on $\operatorname{fmax}(d, n ; j)$ - the maximum number of $j$-faces that a cs $d$-polytope on $n$ vertices can have.

Theorem 1.3 If $d$ is a fixed even number and $n \rightarrow \infty$, then

$$
1-\frac{1}{d-1}+o(1) \leq \frac{\operatorname{fmax}(d, n ; 1)}{\left(\begin{array}{l}
n \\
2
\end{array}\right)} \leq 1-\frac{1}{2^{d}}+o(1)
$$

Theorem 1.4 If $d=2 k$ is a fixed even number, $j \leq k-1$, and $n \rightarrow \infty$, then

$$
c_{j}(d)+o(1) \leq \frac{\operatorname{fmax}(d, n ; j)}{\left(\begin{array}{c}
n \\
j+1
\end{array}\right)} \leq 1-\frac{1}{2^{d}}+o(1),
$$

where $c_{j}(d)$ is a positive constant.

Some discussion is in order. Recall that the cyclic polytope is $\lfloor d / 2\rfloor$-neighborly, that is, for all $j \leq\lfloor d / 2\rfloor$, every $j$ vertices of $C_{d}(n)$ form the vertex set of a face. Since $C_{d}(n)$ is a simplicial polytope, its neighborliness implies that $f_{j}(C(d, n))=\left(\begin{array}{c}n \\ j+1\end{array}\right)$ for $j<\lfloor d / 2\rfloor$. Now if $P$ is a centrally symmetric polytope on $n$ vertices then no two of its antipodal vertices are connected by an edge, and so $f_{1}(P) \leq\left(\begin{array}{l}n \\ 2\end{array}\right)-\frac{n}{2}$. In fact, as was recently shown by Linial and the second author [15], this inequality is strict as long as $n>2^{d}$. This leads one to wonder how big the gap between $\operatorname{fmax}(d, n ; 1)$ and $\left(\begin{array}{l}n \\ 2\end{array}\right)$ is. Theorems 1.3 and 1.4 (see also Propositions 2.1 and 2.2 below) provide (partial) answers to those questions. In Sect. 7.3, we discuss several available lower bounds for $c_{j}(d)$.

Let us fix an even dimension $d=2 k$ and let $X \subset \mathbb{S}^{1}$ be a set of $n$ equally spaced points, where $n$ is an even number. We conjecture that for every integer $j \leq k-1$

$$
\limsup _{n \rightarrow+\infty} \frac{f_{j}\left(\mathcal{B}_{2 k}(X)\right)}{\left(\begin{array}{c}
n \\
j+1
\end{array}\right)}=\limsup _{n \rightarrow+\infty} \frac{\operatorname{fmax}(d, n ; j)}{\left(\begin{array}{c}
n \\
j+1
\end{array}\right)} .
$$

It is also worth mentioning that recently there has been a lot of interest in the problems surrounding neighborliness and face numbers of cs polytopes in connection to statistics and error-correcting codes, see [7-9, 18]. In particular, it was proved in [9] that for large $n$ and $d$, if $j$ is bigger than a certain threshold value, then the ratio between the expected number of $j$-faces of a random cs $d$-polytope with $n$ vertices and $\left(\begin{array}{c}n \\ j+1\end{array}\right)$ is smaller than $1-\epsilon$ for some positive constant $\epsilon$. The upper bound part of Theorem 1.4 provides a real reason for this phenomenon: the expected number of $j$-faces is "small" because the $j$ th face number of every cs polytope is "small".

In 1980s, in an attempt to come up with a centrally symmetric variation of cyclic polytopes, Björner [3] considered convex hulls of certain symmetric sets of points chosen on the odd-moment curve

$$
\mathrm{OM}_{m}(t)=\left(t, t^{3}, t^{5}, \ldots, t^{2 m-1}\right), \quad \mathrm{OM}_{m}(t) \in \mathbb{R}^{m} \quad \text { for } t \in \mathbb{R} .
$$

The authors are not aware of any results similar to Theorems 1.1 and 1.2 for such polytopes. The curves $\mathrm{OM}_{2 k} \subset \mathbb{R}^{2 k}$ and $\mathrm{SM}_{2 k} \subset \mathbb{R}^{2 k}$ behave differently with respect 
to the affine structure on $\mathbb{R}^{2 k}$ : there are affine hyperplanes in $\mathbb{R}^{2 k}$ intersecting $\mathrm{OM}_{2 k}$ in as many as $4 k-1$ points while any affine hyperplane in $\mathbb{R}^{2 k}$ intersects $\mathrm{SM}_{2 k}$ in at most $4 k-2$ points, cf. Sect. 3.3. In contrast, the ordinary and trigonometric moment curves in even dimensions behave quite similarly to each other.

As the anonymous referee pointed out to the authors, $\mathrm{SM}_{2 k}$ is an algebraic curve of degree $4 k-2$ (it is rationalized by the substitution $s=\tan (t / 2)$ ) and $4 k-2$ is the minimum degree that an irreducible centrally symmetric algebraic curve in $\mathbb{R}^{2 k}$ not lying in an affine hyperplane can have. (Note that the degree of $\mathrm{OM}_{2 k}$ is $4 k-1$.)

The structure of the paper is as follows. In Sect. 2 we prove the upper bound parts of Theorems 1.3 and 1.4. In Sect. 3 we discuss bicyclic polytopes and their relationship to non-negative trigonometric polynomials and self-inversive polynomials. Section 4 contains new short proofs of results originally due to Smilansky on the faces of 4-dimensional bicyclic polytopes. It serves as a warm-up for Sects. 5 and 6 in which we prove Theorems 1.1 and 1.2 as well as the lower bound parts of Theorems 1.3 and 1.4. We discuss 2 -faces of $\mathcal{B}_{6}$, values of $\operatorname{fmax}(2 k, n ; j)$ for $j \geq k$, and lower bounds for constants $c_{j}(d)$ of Theorem 1.4 in Sect. 7, where we also state several open questions.

\section{Upper Bounds on the Face Numbers}

The goal of this section is to prove the upper bound parts of Theorems 1.3 and 1.4. The proof uses a volume trick similar to the one utilized in the proof of the DanzerGrünbaum theorem on the number of vertices of antipodal polytopes [6] and more recently in [15, Theorem 1], where it was used to estimate maximal possible neighborliness of cs polytopes.

The upper bound part of Theorem 1.3 is an immediate consequence of the following more precise result on the number of edges of a centrally symmetric polytope.

Proposition 2.1 Let $P \subset \mathbb{R}^{d}$ be a cs $d$-polytope on $n$ vertices. Then

$$
f_{1}(P) \leq \frac{n^{2}}{2}\left(1-2^{-d}\right)
$$

Proof Let $V$ be the set of vertices of $P$. For every vertex $u$ of $P$ we define

$$
P_{u}:=P+u \subset 2 P
$$

to be a translate of $P$, where "+" denotes the Minkowski addition. We claim that if the polytopes $P_{u}$ and $P_{v}$ have intersecting interiors then the vertices $u$ and $-v$ are not connected by an edge. (Note that this includes the case of $u=v$, since clearly $\operatorname{int}\left(P_{v}\right) \cap \operatorname{int}\left(P_{v}\right) \neq \varnothing$ and $(v,-v)$ is not an edge of $P$.) Indeed, the assumption $\operatorname{int}\left(P_{u}\right) \cap \operatorname{int}\left(P_{v}\right) \neq \varnothing$ implies that there exist $x, y \in \operatorname{int}(P)$ such that $x+u=y+v$, or equivalently, that $(y-x) / 2=(u-v) / 2$. Since $P$ is centrally symmetric, and $x, y \in \operatorname{int}(P)$, the point $q:=(y-x) / 2$ is an interior point of $P$. As $q$ is also the barycenter of the line segment connecting $u$ and $-v$, this line segment is not an edge of $P$. 
Now normalize the Lebesgue measure $d x$ in $\mathbb{R}^{d}$ in such a way that $\operatorname{vol}(2 P)=1$ and hence

$$
\operatorname{vol}(P)=\operatorname{vol}\left(P_{u}\right)=2^{-d} \text { for all } u \in V .
$$

For a set $A \subset \mathbb{R}^{d}$, let $[A]: \mathbb{R}^{d} \rightarrow \mathbb{R}$ be the indicator of $A$, that is, $[A](x)=1$ for $x \in A$ and $[A](x)=0$. Define

$$
h=\sum_{u \in V}\left[\operatorname{int} P_{u}\right]
$$

Then

$$
\int_{2 P} h d x=n 2^{-d}
$$

and hence by the Hölder inequality

$$
\int_{2 P} h^{2} d x \geq n^{2} 2^{-2 d}
$$

On the other hand, the first paragraph of the proof implies that

$$
\int_{2 P} h^{2}(x) d x=\sum_{u, v \in V} \operatorname{vol}\left(P_{u} \cap P_{v}\right) \leq n 2^{-d}+2\left(\left(\begin{array}{l}
n \\
2
\end{array}\right)-f_{1}(P)\right) 2^{-d},
$$

and the statement follows.

As a corollary, we obtain the following upper bound on the number $f_{j}(P)$ of $j$-dimensional faces for any $1 \leq j \leq(d-2) / 2$. This upper bound implies the upper bound part of Theorem 1.4.

Proposition 2.2 Let $P \subset \mathbb{R}^{d}$ be a cs $d$-polytope with $n$ vertices, and let $j \leq$ $(d-2) / 2$. Then

$$
f_{j}(P) \leq \frac{n}{n-1}\left(1-2^{-d}\right)\left(\begin{array}{c}
n \\
j+1
\end{array}\right)
$$

Proof We rely on Proposition 2.1 and two additional results.

The first result is an adaptation of the well-known perturbation argument, see, for example, [11, Sect. 5.2], to the centrally symmetric situation. Namely, we claim that for every cs $d$-polytope $P$ there exists a simplicial cs $d$-polytope $Q$ such that $f_{0}(P)=$ $f_{0}(Q)$ and $f_{j}(P) \leq f_{j}(Q)$ for all $1 \leq j \leq d-1$. The polytope $Q$ is obtained from $P$ by pulling the vertices of $P$ in a generic way but so as to preserve the symmetry. The proof is completely similar to that of [11, Sect. 5.2] and hence is omitted.

The second result states that for every $(d-1)$-dimensional simplicial complex $K$ with $n$ vertices, we have

$$
f_{j}(K) \leq f_{1}(K)\left(\begin{array}{c}
n \\
j+1
\end{array}\right) /\left(\begin{array}{l}
n \\
2
\end{array}\right) \text { for } 1 \leq j \leq d-1 .
$$


The standard double-counting argument goes as follows: every $j$-dimensional simplex of $K$ contains exactly $\left(\begin{array}{c}j+1 \\ 2\end{array}\right)$ edges and every edge of $K$ is contained in at most $\left(\begin{array}{c}n-2 \\ j-1\end{array}\right)$ of the $j$-dimensional simplices of $K$. Hence

$$
f_{j}(K) / f_{1}(K) \leq\left(\begin{array}{c}
n-2 \\
j-1
\end{array}\right) /\left(\begin{array}{c}
j+1 \\
2
\end{array}\right)=\left(\begin{array}{c}
n \\
j+1
\end{array}\right) /\left(\begin{array}{l}
n \\
2
\end{array}\right) .
$$

The statement now follows by Proposition 2.1.

\section{Faces and Polynomials}

In this section, we relate the facial structure of the convex hull $\mathcal{B}_{2 k}$ of the symmetric moment curve (Sect. 1.1) to properties of trigonometric and complex polynomials from particular families.

\subsection{Preliminaries}

A proper face of a convex body $B \subset \mathbb{R}^{2 k}$ is the intersection of $B$ with its supporting hyperplane, that is, the intersection of $B$ with the zero-set of an affine function

$$
A(x)=\alpha_{0}+\alpha_{1} \xi_{1}+\cdots+\alpha_{2 k} \xi_{2 k} \quad \text { for } x=\left(\xi_{1}, \ldots, \xi_{2 k}\right)
$$

that satisfies $A(x) \geq 0$ for all $x \in B$.

A useful observation is that $\mathcal{B}_{2 k}$ remains invariant under a one-parametric group of rotations that acts transitively on $\mathrm{SM}_{2 k}\left(\mathbb{S}^{1}\right)$. Such a rotation is represented by a $2 k \times 2 k$ block-diagonal matrix with the $j$ th block being

$$
\left(\begin{array}{cc}
\cos (2 j-1) \tau & \sin (2 j-1) \tau \\
-\sin (2 j-1) \tau & \cos (2 j-1) \tau
\end{array}\right)
$$

for $\tau \in \mathbb{R}$. If $\left\{t_{1}, \ldots, t_{s}\right\} \subset \mathbb{S}^{1}$ are distinct points such that

$$
\operatorname{conv}\left(\mathrm{SM}_{2 k}\left(t_{1}\right), \ldots, \mathrm{SM}_{2 k}\left(t_{s}\right)\right)
$$

is a face of $\mathcal{B}_{2 k}$ and points $t_{1}^{\prime}, \ldots, t_{s}^{\prime} \in \mathbb{S}^{1}$ are obtained from $t_{1}, \ldots, t_{s}$ by the rotation $t_{i}^{\prime}=t_{i}+\tau$ for $i=1, \ldots, s$ of $\mathbb{S}^{1}$, then

$$
\operatorname{conv}\left(\mathrm{SM}_{2 k}\left(t_{1}^{\prime}\right), \ldots, \mathrm{SM}_{2 k}\left(t_{s}^{\prime}\right)\right)
$$

is a face of $\mathcal{B}_{2 k}$ as well.

Finally, we note that the natural projection $\mathbb{R}^{2 k} \rightarrow \mathbb{R}^{2 k^{\prime}}$ for $k^{\prime}<k$ that erases the last $2 k-2 k^{\prime}$ coordinates maps $\mathcal{B}_{2 k}$ onto $\mathcal{B}_{2 k^{\prime}}$ and $\mathcal{B}_{2 k}(X)$ onto $\mathcal{B}_{2 k^{\prime}}(X)$. Hence, if for some sets $Y \subset X \subset \mathbb{S}^{1}$ the set $\operatorname{conv}\left(\operatorname{SM}_{2 k^{\prime}}(Y)\right)$ is a face of $\mathcal{B}_{2 k^{\prime}}(X)$, then $\operatorname{conv}\left(\operatorname{SM}_{2 k}(Y)\right)$ is a face of $\mathcal{B}_{2 k}(X)$. We call a face of $\mathcal{B}_{2 k}$ an old face if it is an inverse image of a face of $\mathcal{B}_{2 k^{\prime}}$ for some $k^{\prime}<k$, and call it a new face otherwise. 


\subsection{Raked Trigonometric Polynomials}

The value of an affine function $A(x)$ on the symmetric moment curve $\mathrm{SM}_{2 k}$ is represented by a trigonometric polynomial

$$
A(t)=c+\sum_{j=1}^{k} a_{j} \cos (2 j-1) t+\sum_{j=1}^{k} b_{j} \sin (2 j-1) t .
$$

Note that all summands involving the even terms $\sin 2 j t$ and $\cos 2 j t$ except for the constant term vanish from $A(t)$. We refer to such trigonometric polynomials as raked trigonometric polynomials of degree at most $2 k-1$. As before, it is convenient to think of $A(t)$ as defined on $\mathbb{S}^{1}=\mathbb{R} / 2 \pi \mathbb{Z}$.

Admitting, for convenience, the whole body $\mathcal{B}_{2 k}$ and the empty set as faces of $\mathcal{B}_{2 k}$, we obtain the following result.

Lemma 3.1 The faces of $\mathcal{B}_{2 k}$ are defined by raked trigonometric polynomials of degree at most $2 k-1$ that are non-negative on $\mathbb{S}^{1}$. If $A(t)$ is such a polynomial and $\left\{t_{1}, \ldots, t_{s}\right\} \subset \mathbb{S}^{1}$ is the set of its zeroes, then the face of $\mathcal{B}_{2 k}$ defined by $A(t)$ is the convex hull of $\left\{\mathrm{SM}_{2 k}\left(t_{1}\right), \ldots, \mathrm{SM}_{2 k}\left(t_{s}\right)\right\}$.

It is worth noticing that if a polynomial $A(t)$ of Lemma 3.1 has degree smaller than $2 k-1$, then the convex hull of $\left\{\mathrm{SM}_{2 k-2}\left(t_{1}\right), \ldots, \mathrm{SM}_{2 k-2}\left(t_{S}\right)\right\}$ is a face of $\mathcal{B}_{2 k-2}$. Thus all new faces of $\mathcal{B}_{2 k}$ are defined by raked trigonometric polynomials of degree $2 k-1$.

\subsection{Raked Self-Inversive Polynomials}

Let us substitute $z=e^{i t}$ in (1). Using that

$$
\cos (2 j-1) t=\frac{z^{2 j-1}+z^{1-2 j}}{2} \text { and } \sin (2 j-1) t=\frac{z^{2 j-1}-z^{1-2 j}}{2 i}
$$

we can write $A(t)=z^{-2 k+1} D(z)$, where

$$
D(z)=c z^{2 k-1}+\sum_{j=1}^{k} \frac{a_{j}-i b_{j}}{2} z^{2 j+2 k-2}+\sum_{j=1}^{k} \frac{a_{j}+i b_{j}}{2} z^{2 k-2 j}
$$

In other words, $D(z)$ is a polynomial satisfying

$$
D(z)=z^{4 k-2} \overline{D(1 / \bar{z})}
$$

and such that

$$
D(z)=c z^{2 k-1}+\sum_{j=0}^{2 k-1} d_{2 j} z^{2 j}
$$

so that all odd terms with the possible exception of the middle term vanish. We note that (2) is equivalent to $\overline{d_{2 j}}=d_{4 k-2-2 j}$ for $j=0, \ldots, 2 k-1$. 
The polynomials $D(z)$ satisfying (2) are well studied and known in the literature by the name self-inversive polynomials, see for instance [19, Chap. 7]). (Some sources require that a self-inversive polynomial $D$ satisfies $D(0) \neq 0$, but we do not.) In analogy with raked trigonometric polynomials, we refer to polynomials $D$ satisfying both (2) and (3) as raked self-inversive polynomials. We note that any polynomial $D(z)$ satisfying (2) and (3) gives rise to a raked trigonometric polynomial $A(t)$ of degree at most $2 k-1$ such that $A(t)=z^{-2 k+1} D(z)$ for $z=e^{i t}$. Furthermore, the multiplicity of a root $t$ of $A$ is equal to the multiplicity of the $\operatorname{root} z=e^{i t}$ of $D$. Hence we obtain the following restatement of Lemma 3.1.

Lemma 3.2 The faces of $\mathcal{B}_{2 k}$ are defined by raked self-inversive polynomials $D(z)$ that satisfy (2) and (3) and all of whose roots of modulus one have even multiplicities. If $D(z)$ is such a polynomial and $\left\{e^{i t_{1}}, \ldots, e^{i t_{s}}\right\}$ is the set of its roots of modulus 1 , then the face of $\mathcal{B}_{2 k}$ defined by $D(z)$ is the convex hull of $\left\{\operatorname{SM}_{2 k}\left(t_{1}\right), \ldots, \mathrm{SM}_{2 k}\left(t_{s}\right)\right\}$.

Clearly, new faces of $\mathcal{B}_{2 k}$ are defined by polynomials of degree $4 k-2$, see Sect. 3.1.

Let $D(z)$ be a polynomial satisfying (2), and let

$$
M=\left\{\zeta_{1}, \ldots, \zeta_{1}, \zeta_{2}, \ldots, \zeta_{2}, \ldots, \zeta_{s}, \ldots, \zeta_{s}\right\}
$$

be the multiset of all roots of $D$ where each root is listed the number of times equal to its multiplicity. We note that if $\operatorname{deg} D=4 k-2$ then $0 \notin M$ and $|M|=4 k-2$. We need a straightforward characterization of the raked self-inversive polynomials in terms of their zero multisets $M$.

Lemma 3.3 A multiset $M \subset \mathbb{C} \backslash\{0\}$ of size $|M|=4 k-2$ is the multiset of roots of a raked self-inversive polynomial of degree $4 k-2$ if and only if the following conditions (a) and (b) are satisfied.

(a) We have

$$
\bar{M}=M^{-1},
$$

that is, $\zeta \in M$ if and only if $\bar{\zeta}^{-1} \in M$ and the multiplicities of $\zeta$ and $\bar{\zeta}^{-1}$ in $M$ (b) are equal, and

$$
\sum_{\zeta \in M} \zeta^{2 j-1}=0 \quad \text { for } j=1, \ldots, k-1
$$

Proof It is known and not hard to see that $M$ is the zero-multiset of a self-inversive polynomial if and only if $M^{-1}=\bar{M}[19$, p. 149, 228]. Indeed, if $D(0) \neq 0$ then (2) implies $\bar{M}=M^{-1}$. Conversely, suppose that $M$ is the multiset satisfying $\bar{M}=M^{-1}$ and such that $|M|=4 k-2$. Then

$$
\prod_{\zeta \in M}|\zeta|=1
$$


and hence we can choose numbers $a_{\zeta}$ such that

$$
\prod_{\zeta \in M} \frac{\bar{a}_{\zeta}}{a_{\zeta}}=\prod_{\zeta \in M}(-\zeta)
$$

Then the polynomial

$$
D(z)=\prod_{\zeta \in M} a_{\zeta}(z-\zeta)
$$

satisfies (2).

Let

$$
s_{m}=\sum_{\zeta \in M} \zeta^{m} \quad \text { and let } D(z)=\sum_{m=0}^{4 k-2} d_{4 k-2-m} z^{m} .
$$

Using Newton's formulas to express elementary symmetric functions in terms of power sums, we obtain

$$
m d_{m}+\sum_{j=1}^{m} s_{j} d_{m-j}=0 \quad \text { for } m=1,2, \ldots, 4 k-2 .
$$

Hence we conclude that

$$
d_{1}=d_{3}=\cdots=d_{2 k-3}=0 \quad \text { if and only if } \quad s_{1}=s_{3}=\cdots=s_{2 k-3}=0,
$$

which completes the proof.

Note that even if a raked self-inversive polynomial $D$ has 0 as its root, it must still satisfy $\sum_{\zeta \in M} \zeta^{2 j-1}=0$, for all $1 \leq j \leq k-1$, where $M$ is the multiset of all roots of $D$.

We conclude this section with the description of a particular family of faces of $\mathcal{B}_{2 k}$.

\subsection{Some Simplicial Faces of $\mathcal{B}_{2 k}$}

Let

$$
A(t)=1-\cos ((2 k-1) t)
$$

Clearly, $A(t)$ is a raked trigonometric polynomial and $A(t) \geq 0$ for all $t \in \mathbb{S}^{1}$. Moreover, $A(t)=0$ at the $2 k-1$ points

$$
\tau_{j}=\frac{2 \pi j}{2 k-1} \quad \text { for } j=1, \ldots, 2 k-1
$$

on the circle $\mathbb{S}^{1}$, which form the vertex set of a regular $(2 k-1)$-gon. By Lemma 3.1 the set

$$
\Delta_{0}=\operatorname{conv}\left(\mathrm{SM}_{2 k}\left(\tau_{1}\right), \ldots, \mathrm{SM}_{2 k}\left(\tau_{2 k-1}\right)\right)
$$

is a face of $\mathcal{B}_{2 k}$. 
One can observe that $\Delta_{0}$ is a $(2 k-2)$-dimensional simplex. To prove that, we have to show that the points $\operatorname{SM}_{2 k}\left(\tau_{1}\right), \ldots, \mathrm{SM}_{2 k}\left(\tau_{2 k-1}\right)$ are affinely independent, or, equivalently, that the affine hyperplanes in $\mathbb{R}^{2 k}$ passing through these points form a one-parametric family (topologically, this set of hyperplanes is a circle). As in Sect. 3.3, those hyperplanes correspond to non-zero complex polynomials $D(z)$ that satisfy (2) and (3) and for which $D\left(e^{i \tau_{j}}\right)=0$ for $j=1, \ldots, 2 k-1$. Hence for each such $D$ we must have

$$
D(z)=\left(z^{2 k-1}-1\right) D_{1}(z),
$$

for some polynomial $D_{1}(z)$ with $\operatorname{deg} D_{1}=s \leq 2 k-1$. Moreover, it follows from (2) that

$$
D_{1}(z)=-z^{2 k-1} \overline{D_{1}(1 / \bar{z})} .
$$

Let $M$ be the multiset of all roots of $D$ and let $M_{1}$ be the multiset of all roots of $D_{1}$. Applying Lemma 3.3, we deduce that

$$
\sum_{\zeta \in M_{1}} \zeta^{2 j-1}=\sum_{\zeta \in M} \zeta^{2 j-1}=0 \quad \text { for } j=1, \ldots, k-1 .
$$

Now, as in the proof of Lemma 3.3 we conclude that the odd-power coefficients of $z$ in $D_{1}$ are zeros except, possibly, for that of $z^{2 k-1}$. In view of (4), all other coefficients of $D_{1}$ except, possibly, the constant term, must be zeros as well. Summarizing, $D_{1}(z)=$ $\alpha z^{2 k-1}-\bar{\alpha}$ for some $\alpha \in \mathbb{C} \backslash\{0\}$. Normalizing $|D(0)|=1$ we get a one-parametric family of polynomials $D(z)=\left(z^{2 k-1}-1\right)\left(\alpha z^{2 k-1}-\bar{\alpha}\right),|\alpha|=1$, that corresponds to the set of affine hyperplanes in $\mathbb{R}^{2 k}$ passing through the vertices of $\Delta_{0}$.

Therefore $\Delta_{0}$ is indeed a $(2 k-2)$-dimensional simplex. Furthermore, we have a one-parametric family of simplicial faces

$$
\Delta_{\tau}=\operatorname{conv}\left(\mathrm{SM}_{2 k}\left(\tau_{1}+\tau\right), \ldots, \mathrm{SM}_{2 k}\left(\tau_{2 k-1}+\tau\right)\right) \quad \text { for } 0 \leq \tau<2 \pi
$$

of $\mathcal{B}_{2 k}$. The dimension of the boundary of $\mathcal{B}_{2 k}$ is $(2 k-1)$, so this one-parametric family of simplices covers a "chunk" of the boundary of $\mathcal{B}_{2 k}$. Borrowing a term from the polytope theory, we may call $\Delta_{\tau}$ a "ridge" of $\mathcal{B}_{2 k}$. As follows from Sect. 4, for $k=2$ the simplices $\Delta_{\tau}$ are the only ridges of $\mathcal{B}_{2 k}$.

\section{The Faces of $\mathcal{B}_{4}$}

In this section we provide a complete characterization of the faces of $\mathcal{B}_{4}$. This result is not new, it was proved by Smilansky [20] who also described the facial structure of the convex hull of the more general curve $(\cos p t, \sin p t, \cos q t, \sin q t)$, where $p$ and $q$ are any positive integers. Our proof serves as a warm-up for the following section where we discuss the edges of $\mathcal{B}_{2 k}$ for $k>2$. 
Theorem 4.1 [20] The proper faces of $\mathcal{B}_{4}$ are

(0) the 0-dimensional faces (vertices)

$$
\mathrm{SM}_{4}(t), \quad t \in \mathbb{S}^{1}
$$

(1) the 1-dimensional faces (edges)

$$
\left[\mathrm{SM}_{4}\left(t_{1}\right), \mathrm{SM}_{4}\left(t_{2}\right)\right]
$$

where $t_{1} \neq t_{2}$ are the endpoints of an arc of $\mathbb{S}^{1}$ of length less than $2 \pi / 3$; and (2) the 2-dimensional faces (equilateral triangles)

$$
\Delta_{t}=\operatorname{conv}\left(\mathrm{SM}_{4}(t), \mathrm{SM}_{4}(t+2 \pi / 3), \mathrm{SM}_{4}(t+4 \pi / 3)\right), \quad t \in \mathbb{S}^{1} \text {. }
$$

Proof We use Lemma 3.2. A face of $\mathcal{B}_{4}$ is determined by a raked self-inversive polynomial $D$ of degree at most 6 . Such a polynomial $D$ has at most 3 roots on the circle $\mathbb{S}^{1}$, each having an even multiplicity. Furthermore, by Lemma 3.3, the sum of all the roots of $D$ is 0 . Therefore, we have the following three cases.

Polynomial $D$ has 3 double roots $\zeta_{1}=e^{i t_{1}}, \zeta_{2}=e^{i t_{2}}, \zeta_{3}=e^{i t_{3}}$. Since $\zeta_{1}+\zeta_{2}+$ $\zeta_{3}=0$, the points $t_{1}, t_{2}, t_{3} \in \mathbb{S}^{1}$ form the vertex set of an equilateral triangle, and we obtain the 2-dimensional face defined in Part (2).

Polynomial $D$ has two double roots $\zeta_{1}=e^{i t_{1}}, \zeta_{2}=e^{i t_{2}}$, and a pair of simple roots $\zeta$ and $\bar{\zeta}^{-1}$ with $|\zeta| \neq 1$. Applying a rotation, if necessary, we may assume without loss of generality that $t_{1}=-t_{2}=t$. Since we must have

$$
\zeta+\bar{\zeta}^{-1}+2 e^{i t}+2 e^{-i t}=0
$$

we conclude that $\zeta \in \mathbb{R}$. Hence the equation reads

$$
\zeta+\zeta^{-1}=-4 \cos t \quad \text { for some } \zeta \in \mathbb{R},|\zeta| \neq 1
$$

If $|\cos t|>1 / 2$ then the solutions $\zeta, \zeta^{-1}$ of this equation are indeed real and satisfy $|\zeta|,\left|\zeta^{-1}\right| \neq 1$. If $|\cos t| \leq 1 / 2$ then $\left\{\zeta, \zeta^{-1}\right\}$ is a pair of complex conjugate numbers satisfying $|\zeta|=\left|\zeta^{-1}\right|=1$. Therefore, the interval $\left[\mathrm{SM}_{4}(-t), \mathrm{SM}_{4}(t)\right]$ is a face of $\mathcal{B}_{4}$ if and only if $-\pi / 3<t<\pi / 3$ or $2 \pi / 3<t<4 \pi / 3$, so we obtain the 1-dimensional faces as in Part (1).

Finally, Lemma 3.1 applied to $A(t)=1-\cos (\tau-t)$ yields that $\operatorname{SM}_{4}(\tau)$ is a 0 -dimensional face (vertex) of $\mathcal{B}_{4}$ for every $\tau \in \mathbb{S}^{1}$, which concludes the proof.

\section{Edges of $\mathcal{B}_{2 k}$}

In this section we prove Theorems 1.1 and 1.3. Our main tool is a certain deformation of simplicial faces of $\mathcal{B}_{2 k}$, cf. Sect. 3.4. 


\subsection{Deformation}

Let $M$ be a finite multiset of non-zero complex numbers such that $M=M^{-1}$. In other words, for every $\zeta \in M$ we have $\zeta^{-1} \in M$ and the multiplicities of $\zeta$ and $\zeta^{-1}$ in $M$ are equal. In addition, we assume that the multiplicities of 1 and -1 in $M$ are even, possibly 0 . For every $\lambda \in \mathbb{R} \backslash\{0\}$ we define a multiset $M_{\lambda}$, which we call a deformation of $M$, as follows.

We think of $M$ as a multiset of unordered pairs $\left\{\zeta, \zeta^{-1}\right\}$. For each such pair, we consider the equation

$$
z+z^{-1}=\lambda\left(\zeta+\zeta^{-1}\right)
$$

We let $M_{\lambda}$ to be the multiset consisting of the pairs $\left\{z, z^{-1}\right\}$ of solutions of (5) as $\left\{\zeta, \zeta^{-1}\right\}$ range over $M$. Clearly, $\left|M_{\lambda}\right|=|M|$ and $M_{\lambda}^{-1}=M_{\lambda}$. In addition, if $\bar{M}=M$ then $\overline{M_{\lambda}}=M_{\lambda}$, since $\lambda$ in (5) is real.

Our interest in the deformation $M \mapsto M_{\lambda}$ is explained by the following lemma.

Lemma 5.1 Let $D(z)$ be a raked self-inversive polynomial of degree $4 k-2$ with real coefficients and such that $D(0) \neq 0$. Let $M$ be the multiset of all roots of $D$ and suppose that both 1 and -1 have an even, possibly 0 , multiplicity in $M$. Then, for every real $\lambda \neq 0$, the deformation $M_{\lambda}$ of $M$ is the multiset of all roots of a raked self-inversive polynomial $D_{\lambda}(z)$ of degree $4 k-2$ with real coefficients.

Proof We use Lemma 3.3. Since $D$ has real coefficients, we have $M=\bar{M}$, so by Lemma 3.3, we have $M=M^{-1}$ as well. Then $M_{\lambda}=M_{\lambda}^{-1}$ and $M_{\lambda}=\overline{M_{\lambda}}$, so $M_{\lambda}$ is the multiset of the roots of a self-inversive real polynomial $D_{\lambda}$ of degree $4 k-2$. It remains to check that

$$
\sum_{\zeta \in M_{\lambda}} \zeta^{2 j-1}=0 \quad \text { for } j=1, \ldots, k-1 .
$$

We have

$$
\left(x+x^{-1}\right)^{2 n-1}=\sum_{m=1}^{n}\left(\begin{array}{c}
2 n-1 \\
n+m-1
\end{array}\right)\left(x^{2 m-1}+x^{-2 m+1}\right) .
$$

Since by Lemma 3.3

$$
\sum_{\zeta \in M} \zeta^{2 j-1}=\sum_{\zeta \in M} \zeta^{1-2 j}=0 \quad \text { for } j=1, \ldots, k-1,
$$

it follows by (6) that

$$
\sum_{\zeta \in M}\left(\zeta+\zeta^{-1}\right)^{2 j-1}=0 \quad \text { for } j=1, \ldots, k-1 .
$$

Therefore, by (5), we have

$$
\sum_{\zeta \in M_{\lambda}}\left(\zeta+\zeta^{-1}\right)^{2 j-1}=0 \quad \text { for } j=1, \ldots, k-1,
$$


from which by (6) we obtain

$$
\sum_{\zeta \in M_{\lambda}} \zeta^{2 j-1}=\frac{1}{2} \sum_{\zeta \in M_{\lambda}}\left(\zeta^{2 j-1}+\zeta^{-2 j+1}\right)=0 \quad \text { for } j=1, \ldots, k-1,
$$

as claimed. Hence $D_{\lambda}(z)$ is a raked self-inversive polynomial.

To prove Theorem 1.1 we need another auxiliary result.

Lemma 5.2 Let $\alpha, \beta \in \mathbb{S}^{1}$ be such that the interval $\left[\operatorname{SM}_{2 k}(\alpha), \operatorname{SM}_{2 k}(\beta)\right]$ is an edge of $\mathcal{B}_{2 k}$ and let $\alpha^{\prime} \neq \beta^{\prime} \in \mathbb{S}^{1}$ be some other points such that the arc with the endpoints $\alpha^{\prime}, \beta^{\prime}$ is shorter than the arc with the endpoints $\alpha, \beta \in \mathbb{S}^{1}$. Then the interval $\left[\mathrm{SM}_{2 k}\left(\alpha^{\prime}\right), \mathrm{SM}_{2 k}\left(\beta^{\prime}\right)\right]$ is an edge of $\mathcal{B}_{2 k}$.

Proof Because of rotational invariance, we assume, without loss of generality, that $\alpha=\tau$ and $\beta=-\tau$ for some $0<\tau<\pi / 2$. Let $A(t)$ be a raked trigonometric polynomial that defines the edge $\left[\operatorname{SM}_{2 k}(\alpha), \operatorname{SM}_{2 k}(\beta)\right]$, see Lemma 3.1. Hence $A(t) \geq 0$ for all $t \in \mathbb{S}^{1}$ and $A(t)=0$ if and only $t= \pm \tau$. Let $A_{1}(t)=A(t)+A(-t)$. Then $A_{1}(t)$ is a raked trigonometric polynomial such that $A_{1}(t) \geq 0$ for all $t \in \mathbb{S}^{1}$ and $A_{1}(t)=0$ if and only if $t= \pm \tau$. Furthermore, we can write

$$
A_{1}(t)=c+\sum_{j=1}^{k} a_{j} \cos (2 j-1) t
$$

for some real $a_{j}$ and $c$. Moreover, we assume, without loss of generality, that $a_{k} \neq 0$. (Otherwise choose $k^{\prime}$ to be the largest index $j$ with $a_{j} \neq 0$ and project $\mathcal{B}_{2 k}$ onto $\mathcal{B}_{2 k^{\prime}}$, cf. Sect. 3.1.) Hence the polynomial $D(z)$ defined by $A_{1}(t)=z^{-2 k+1} D(z)$ for $z=e^{i t}$, see Sect. 3.3, is a raked self-inversive polynomial of degree $4 k-2$ with real coefficients satisfying $D(0) \neq 0$. Moreover, the only roots of $D(z)$ that lie on the circle $|z|=1$ are $e^{i \tau}$ and $e^{-i \tau}$ and those roots have equal even multiplicities.

Choose an arbitrary $0<\tau^{\prime}<\tau$ and let

$$
\lambda=\frac{\cos \tau^{\prime}}{\cos \tau}>1
$$

Let $D_{\lambda}$ be the raked self-inversive polynomial of degree $4 k-2$ whose existence is established by Lemma 5.1. Since

$$
e^{i \tau^{\prime}}+e^{-i \tau^{\prime}}=\lambda\left(e^{i \tau}+e^{-i \tau}\right)
$$

the numbers $e^{i \tau^{\prime}}$ and $e^{-i \tau^{\prime}}$ are roots of $D_{\lambda}$ of even multiplicity. Moreover, suppose that $z$ is a root of $D_{\lambda}$ such that $|z|=1$. Then $z+z^{-1} \in \mathbb{R}$ and $-2 \leq z+z^{-1} \leq 2$. By (5) and from the fact that $\lambda>1$, it follows that there is a pair $\zeta, \zeta^{-1}$ of roots of $D$ such that $\zeta+\zeta^{-1} \in \mathbb{R}$ and $-2<\left|\zeta+\zeta^{-1}\right|<2$. It follows then that $|\zeta|=\left|\zeta^{-1}\right|=1$, which necessarily yields that $\left\{\zeta, \zeta^{-1}\right\}=\left\{e^{i \tau}, e^{-i \tau}\right\}$, and hence that $\left\{z, z^{-1}\right\}=\left\{e^{i \tau^{\prime}}, e^{-i \tau^{\prime}}\right\}$. Therefore, by Lemma 3.2, $\left[\mathrm{SM}_{2 k}\left(-\tau^{\prime}\right), \mathrm{SM}_{2 k}\left(\tau^{\prime}\right)\right]$ is an edge of $\mathcal{B}_{2 k}$. Using rotational invariance, we infer that $\left[\mathrm{SM}_{2 k}\left(\alpha^{\prime}\right), \mathrm{SM}_{2 k}\left(\beta^{\prime}\right)\right]$ is an edge of $\mathcal{B}_{2 k}$, where points $\alpha^{\prime}, \beta^{\prime}$ are obtained from $\tau^{\prime},-\tau^{\prime}$ by an appropriate rotation of $\mathbb{S}^{1}$. 
We are now ready to complete the proof of Theorem 1.1 .

Proof of Theorem 1.1 In view of Lemma 5.2, it remains to show that one can find an arbitrarily small $\delta>0$ and two points $\alpha, \beta \in \mathbb{S}^{1}$ such that the interval $\left[\mathrm{SM}_{2 k}(\alpha), \mathrm{SM}_{2 k}(\beta)\right]$ is an edge of $\mathcal{B}_{2 k}$ and the length of the arc with the endpoints $\alpha$ and $\beta$ is at least $\frac{2 \pi(k-1)}{2 k-1}-\delta$.

Consider the polynomial

$$
D(z)=\left(z^{2 k-1}-1\right)^{2}=z^{4 k-2}-2 z^{2 k-1}+1 .
$$

Clearly, $D(z)$ is a raked self-inversive polynomial of degree $4 k-2$ and the multiset $M$ of the roots of $D$ consists of all roots of unity of degree $2 k-1$, each with multiplicity 2. In fact, $D(z)$ defines a simplicial face of $\mathcal{B}_{2 k}$, cf. Sect. 3.4. Note that since $\mathcal{B}_{2 k}$ is not polyhedral, a face of a face of $\mathcal{B}_{2 k}$ does not have to be a face of $\mathcal{B}_{2 k}$.

For $\epsilon>0$ we consider the deformation $D_{1+\epsilon}(z)$ of $D(z)$ and its roots, see Lemma 5.1. In view of equation (5), for all sufficiently small $\epsilon>0$, the multiset $M_{1+\epsilon}$ of the roots of $D_{1+\epsilon}$ consists of two positive simple real roots defined by the equation

$$
z+z^{-1}=2(1+\epsilon)
$$

and $2 k-2$ double roots on the unit circle defined by the equation

$$
z+z^{-1}=2(1+\epsilon) \cos \frac{2 \pi j}{2 k-1} \quad \text { for } j=1, \ldots, 2 k-2 .
$$

The first two of these roots are deformations of the double root at 1 (one of them is strictly larger and another one is strictly smaller than number 1), while the other roots are deformations of the remaining $2 k-2$ roots of unity.

For $\epsilon>0$ small enough let $\zeta_{j}(\epsilon)$ denote the deformation of the root

$$
\zeta_{j}=\cos \frac{2 \pi j}{2 k-1}+i \sin \frac{2 \pi j}{2 k-1} \quad \text { for } j=1, \ldots, 2 k-2
$$

that lies close to $\zeta_{j}$, see Fig. 1. Thus we have

$$
\zeta_{j}^{-1}(\epsilon)=\overline{\zeta_{j}}(\epsilon)=\zeta_{2 k-1-j}(\epsilon)
$$

Fig. 1 The roots of unity (black dots) and their deformations (white dots) for $k=3$

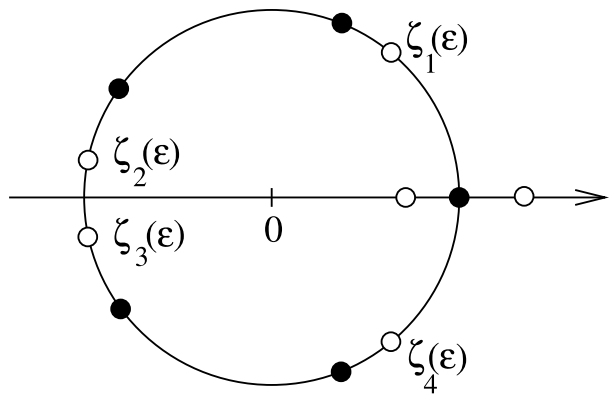


Write

$$
\zeta_{j}(\epsilon)=e^{i \alpha_{j}} \quad \text { where } 0<\alpha_{j}<2 \pi \text { for } j=1, \ldots, 2 k-2 \text {. }
$$

Then

$$
\cos \alpha_{j}=(1+\epsilon) \cos \frac{2 \pi j}{2 k-1},
$$

and hence

$$
\alpha_{j}=\frac{2 \pi j}{2 k-1}-\epsilon \operatorname{ctg} \frac{2 \pi j}{2 k-1}+O\left(\epsilon^{2}\right) .
$$

We now prove that the interval

$$
\left[\mathrm{SM}_{2 k}\left(\alpha_{1}\right), \mathrm{SM}_{2 k}\left(\alpha_{k}\right)\right]
$$

is an edge of $\mathcal{B}_{2 k}$. We obtain this edge as the intersection of two faces of $\mathcal{B}_{2 k}$. The first face is

$$
\operatorname{conv}\left(\mathrm{SM}_{2 k}\left(\alpha_{1}\right), \ldots, \mathrm{SM}_{2 k}\left(\alpha_{2 k-2}\right)\right),
$$

which by Lemmas 3.2 and 5.1 is indeed a face of $\mathcal{B}_{2 k}$. The second face is obtained by a rotation of (9). Namely, consider the clockwise rotation of the circle $|z|=1$ that maps $\zeta_{k-1}(\epsilon)$ onto $\zeta_{1}(\epsilon)$. Because of (7) this rotation also maps $\zeta_{2 k-2}(\epsilon)$ onto $\zeta_{k}(\epsilon)$. Furthermore, for $j=1, \ldots, 2 k-2$ define

$$
\zeta_{j}^{\prime}(\epsilon)=e^{i \alpha_{j}^{\prime}}, \quad \text { where } 0<\alpha_{j}^{\prime}<2 \pi,
$$

as the image under this rotation of $\zeta_{j+k-2}(\epsilon)$ if $j \leq k$, of $\zeta_{j-k-1}(\epsilon)$ if $j>k+1$, and of $\zeta_{k-2}(\epsilon)$ if $j=k+1$. By rotational invariance, see Sect. 3.1,

$$
\operatorname{conv}\left(\mathrm{SM}_{2 k}\left(\alpha_{1}^{\prime}\right), \ldots, \mathrm{SM}_{2 k}\left(\alpha_{2 k-2}^{\prime}\right)\right)
$$

is a face of $\mathcal{B}_{2 k}$ as well.

Using (8), we conclude that

$$
\begin{aligned}
& \alpha_{j}^{\prime}-\alpha_{j}=\epsilon\left(\operatorname{ctg} \frac{2 \pi j}{2 k-1}-\operatorname{ctg} \frac{2 \pi j-3 \pi}{2 k-1}-\operatorname{ctg} \frac{\pi}{2 k-1}-\operatorname{ctg} \frac{2 \pi}{2 k-1}\right)+O\left(\epsilon^{2}\right) \\
& \quad \text { for } 1 \leq j \leq 2 k-2, j \neq k+1
\end{aligned}
$$

and

$$
\alpha_{k+1}^{\prime}=o(1) \quad \text { as } \epsilon \rightarrow 0+
$$

Therefore for a sufficiently small $\epsilon>0$ and $j \neq k+1$, the value of $\alpha_{j}^{\prime}$ is close to and strictly smaller than $\alpha_{j}$ unless $j=1$ or $j=k$, in which case the two values are equal. Furthermore, $\alpha_{k+1}^{\prime} \neq \alpha_{j}$ for all $j$. Thus faces (9) and (10) intersect along the interval

$$
\left[\mathrm{SM}_{2 k}\left(\alpha_{1}\right), \mathrm{SM}_{2 k}\left(\alpha_{k}\right)\right]
$$


and this interval is an edge of $\mathcal{B}_{2 k}$. Since $\alpha_{1}$ and $\alpha_{k}$ are the endpoints of an arc of length

$$
\pi \frac{2 k-2}{2 k-1}-O(\epsilon)
$$

the statement follows.

Proof of Theorem 1.3 The upper bound follows by Proposition 2.1. To prove the lower bound, consider the family of polytopes $\mathcal{B}_{2 k}\left(X_{n}\right)$, where $X_{n} \subset \mathbb{S}^{1}$ is the set of $n$ equally spaced points ( $n$ is even). The lower bound then follows by Theorem 1.1.

\section{Faces of $\mathcal{B}_{2 k}$}

In this section, we prove Theorems 1.2 and 1.4. Theorem 1.2 is deduced from the following proposition.

Proposition 6.1 For every positive integer $k$ there exists a number $\phi_{k}>0$ such that every set of $2 k$ distinct points $t_{1}, \ldots, t_{2 k} \in \mathbb{S}^{1}$ lying on an arc of length at most $\phi_{k}$ is the set of the roots of some raked trigonometric polynomial $A: \mathbb{S}^{1} \rightarrow \mathbb{R}$,

$$
A(t)=c_{0}+\sum_{j=1}^{k} a_{j} \sin (2 j-1) t+\sum_{j=1}^{k} b_{j} \cos (2 j-1) t
$$

To prove Proposition 6.1, we establish first that the curve $\mathrm{SM}_{2 k}(t)$ is nowhere locally flat.

\section{Lemma 6.2 Let}

$$
\mathrm{SM}_{2 k}(t)=(\cos t, \sin t, \cos 3 t, \sin 3 t, \ldots, \cos (2 k-1) t, \sin (2 k-1) t)
$$

be the symmetric moment curve. Then, for every $t \in \mathbb{R}^{1}$, the vectors

$$
\operatorname{SM}_{2 k}(t), \quad \frac{d}{d t} \operatorname{SM}_{2 k}(t), \quad \frac{d^{2}}{d t^{2}} \operatorname{SM}_{2 k}(t), \quad \ldots, \quad \frac{d^{2 k-1}}{d t^{2 k-1}} \operatorname{SM}_{2 k}(t)
$$

are linearly independent.

Proof Because of rotational invariance, it suffices to prove the result for $t=0$. Consider the $2 k$ vectors

$$
\mathrm{SM}_{2 k}(0), \quad \frac{d}{d t} \mathrm{SM}_{2 k}(0), \quad \ldots, \quad \frac{d^{2 k-1}}{d t^{2 k-1}} \operatorname{SM}_{2 k}(0),
$$

that is, the vectors

$$
\begin{aligned}
a_{j} & =(-1)^{j}\left(1,0,3^{2 j}, 0, \ldots, 0,(2 k-1)^{2 j}\right) \text { and } \\
b_{j} & =(-1)^{j}\left(0,1,0,3^{2 j+1}, \ldots,(2 k-1)^{2 j+1}, 0\right)
\end{aligned}
$$


for $j=0, \ldots, k-1$. It is easy to see that the set of vectors $\left\{a_{j}, b_{j}: j=0, \ldots\right.$, $k-1\}$ is linearly independent if and only if both sets of vectors $\left\{a_{j}, j=0, \ldots, k-1\right\}$ and $\left\{b_{j}: j=0, \ldots, k-1\right\}$ are linearly independent. And indeed, the odd-numbered coordinates of $(-1)^{j} a_{j}$ form the $k \times k$ Vandermonde matrix

$$
\left(\begin{array}{ccccc}
1 & 1 & 1 & \ldots & 1 \\
1 & 3^{2} & 5^{2} & \ldots & (2 k-1)^{2} \\
\ldots & \ldots & \ldots & \ldots & \ldots \\
1 & 3^{2 k-2} & 5^{2 k-2} & \ldots & (2 k-1)^{2 k-2}
\end{array}\right)
$$

while the even-numbered coordinates of $(-1)^{j} b_{j}$ form the $k \times k$ Vandermonde matrix

$$
\left(\begin{array}{ccccc}
1 & 3 & 5 & \ldots & (2 k-1) \\
1 & 3^{3} & 5^{3} & \ldots & (2 k-1)^{3} \\
\ldots & \ldots & \ldots & \ldots & \ldots \\
1 & 3^{2 k-1} & 5^{2 k-1} & \ldots & (2 k-1)^{2 k-1}
\end{array}\right)
$$

Hence the statement follows.

Next, we establish a curious property of zeros of raked trigonometric polynomials.

\section{Lemma 6.3 Let}

$$
A(t)=c_{0}+\sum_{j=1}^{k} a_{j} \sin (2 j-1) t+\sum_{j=1}^{k} b_{j} \cos (2 j-1) t
$$

be a raked trigonometric polynomial $A: \mathbb{S}^{1} \rightarrow \mathbb{R}$ that is not identically 0 . Suppose that $A$ has $2 k$ distinct roots in an arc $\Omega \subset \mathbb{S}^{1}$ of length less than $\pi$. Then, if $A$ has yet another root on $\mathbb{S}^{1}$, that root must lie in the arc $\Omega+\pi$.

Proof Consider the derivative of $A(t)$,

$$
A^{\prime}(t)=\sum_{j=1}^{k} a_{j}(2 j-1) \cos (2 j-1) t-\sum_{j=1}^{k} b_{j}(2 j-1) \sin (2 j-1) t,
$$

as a map from $\mathbb{S}^{1}$ to $\mathbb{R}$. Substituting $z=e^{i t}$, we can write

$$
A^{\prime}(t)=\frac{1}{z^{2 k-1}} P(z)
$$

where $P(z)$ is a polynomial of degree at most $4 k-2$, cf. Sect. 3.3. Hence the total number of the roots of $A^{\prime}$ in $\mathbb{S}^{1}$, counting multiplicities, does not exceed $4 k-2$.

Let $t_{0}, t_{1} \in \Omega$ be the roots of $A$ closest to the endpoints of $\Omega$. By Rolle's theorem, $A^{\prime}$ has at least $2 k-1$ distinct roots between $t_{0}$ and $t_{1}$ in $\Omega$. Since $A^{\prime}(t+\pi)=-A^{\prime}(t)$, we must have another $2 k-1$ distinct roots of $A^{\prime}$ in the arc $\Omega+\pi$ between $t_{0}+\pi$ and $t_{1}+\pi$, see Fig. 2 .

Suppose that $A$ has a root $z \in \mathbb{S}^{1}$ outside of $\Omega \cup(\Omega+\pi)$. Then either $z$ lies in the open arc with the endpoints $t_{0}$ and $t_{1}+\pi$ or $z$ lies in the open arc with the endpoints 
Fig. 2 The roots of $A$ (black dots) and roots of $A^{\prime}$ (white dots)

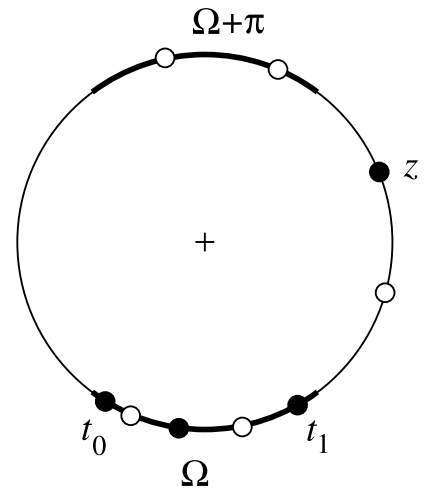

$t_{1}$ and $t_{0}+\pi$. By Rolle's theorem, $A^{\prime}$ has yet another root in $\mathbb{S}^{1}$ between $t_{0}$ on $z$ in the first case, and between $z$ and $t_{1}$ in the second case, which is a contradiction.

We are now ready to prove Proposition 6.1.

Proof of Proposition 6.1 First, we observe that for any $2 k$ points $t_{1}, \ldots, t_{2 k} \in \mathbb{S}^{1}$ there is an affine hyperplane passing through the points $\operatorname{SM}_{2 k}\left(t_{1}\right), \ldots, \mathrm{SM}_{2 k}\left(t_{2 k}\right)$ in $\mathbb{R}^{2 k}$ and hence there is a non-zero raked trigonometric polynomial $A$ such that $A\left(t_{1}\right)=\cdots=A\left(t_{2 k}\right)=0$. Moreover, if $t_{1}, \ldots, t_{2 k}$ are distinct points that lie in an arc $\Omega$ of length less than $\pi$ then the hyperplane is unique. Indeed, if the hyperplane is not unique then the points $\mathrm{SM}_{2 k}\left(t_{1}\right), \ldots, \mathrm{SM}_{2 k}\left(t_{2 k}\right)$ lie in an affine subspace of codimension at least 2 . Therefore, for any point $t_{2 k+1} \in \Omega \backslash\left\{t_{1}, \ldots, t_{2 k}\right\}$ there is an affine hyperplane passing through $\mathrm{SM}_{2 k}\left(t_{1}\right), \ldots, \mathrm{SM}_{2 k}\left(t_{2 k+1}\right)$ and hence there is a raked trigonometric polynomial that has $2 k+1$ roots in $\Omega$ and is not identically 0 , contradicting Lemma 6.3.

Suppose now that no matter how small $\phi_{k}>0$ is, there is always an arc $\Omega \subset \mathbb{S}^{1}$ of length at most $\phi_{k}$ and a non-zero raked trigonometric polynomial $A$ of degree $2 k-1$ that has $2 k$ distinct roots in $\Omega$ and at least one more root elsewhere in $\mathbb{S}^{1}$. By Lemma 6.3, that remaining root must lie in the arc $\Omega+\pi$. In other words, for any positive integer $n$ there exists an arc $\Omega_{n} \subset \mathbb{S}^{1}$ of length at most $1 / n$ and an affine hyperplane $H_{n}$ which intersects $\operatorname{SM}_{2 k}\left(\Omega_{n}\right)$ in $2 k$ distinct points and also intersects the set $\operatorname{SM}_{2 k}\left(\Omega_{n}+\pi\right)$. The set of all affine hyperplanes intersecting the compact set $\mathrm{SM}_{2 k}\left(\mathbb{S}^{1}\right)$ is compact in the natural topology; for example if we view the set of affine hyperplanes in $\mathbb{R}^{2 k}$ as a subset of the Grassmannian of all (linear) hyperplanes in $\mathbb{R}^{2 k+1}$. Therefore, the sequence of hyperplanes $H_{n}$ has a limit hyperplane $H$. By Lemma 6.2 , the affine hyperplane $H$ is the $(2 k-1)$ th order tangent hyperplane to $\mathrm{SM}_{2 k}\left(\mathbb{S}^{1}\right)$ at some point $\mathrm{SM}_{2 k}\left(t_{0}\right)$ where $t_{0}$ is a limit point of the arcs $\Omega_{n}$. Also, $H$ passes through the point $-\mathrm{SM}_{2 k}\left(t_{0}\right)$. The corresponding trigonometric polynomial $A(t)$ is a raked polynomial of degree at most $2 k-1$ that is not identically 0 and has two roots $t_{0}$ and $t_{0}+\pi$ with the multiplicity of $t_{0}$ being at least $2 k$.

Let

$$
A(t)=c_{0}+\sum_{j=1}^{k} a_{j} \sin (2 j-1) t+\sum_{j=1}^{k} b_{j} \cos (2 j-1) t
$$


Since $A\left(t_{0}\right)=A\left(t_{0}+\pi\right)=0$ we conclude that $c_{0}=0$. This, however, contradicts Lemma 6.2 since the non-zero $2 k$-vector

$$
\left(b_{1}, a_{1}, b_{2}, a_{2}, \ldots, b_{k}, a_{k}\right)
$$

turns out to be orthogonal to vectors

$$
\mathrm{SM}_{2 k}\left(t_{0}\right), \quad \frac{d}{d t} \mathrm{SM}_{2 k}\left(t_{0}\right), \quad \ldots, \quad \frac{d^{2 k-1}}{d t^{2 k-1}} \mathrm{SM}_{2 k}\left(t_{0}\right) .
$$

Proof of Theorem 1.2 Let $\phi_{k}>0$ be the number whose existence is established in Proposition 6.1. Given $k$ distinct points $t_{1}, \ldots, t_{k}$ lying on an arc of length at most $\phi_{k}$, we must present a raked trigonometric polynomial $A$ that has roots of multiplicity two at $t_{1}, \ldots, t_{k}$ and no other roots on the circle. In geometric terms, we must present an affine hyperplane that is the first order tangent to the points $\operatorname{SM}_{2 k}\left(t_{1}\right), \ldots, \mathrm{SM}_{2 k}\left(t_{k}\right)$ and does not intersect $\mathrm{SM}_{2 k}\left(\mathbb{S}^{1}\right)$ anywhere else. As in the proof of Proposition 6.1, such a hyperplane is obtained as a limit of the affine hyperplanes that for every $j=$ $1, \ldots, k$ intersect $\mathrm{SM}_{2 k}\left(\mathbb{S}^{1}\right)$ at two distinct points converging to $t_{j}$.

Proof of Theorem 1.4 The upper bound follows by Proposition 2.2. To prove the lower bound, consider the family of polytopes $\mathcal{B}_{2 k}\left(X_{n}\right)$, where $X_{n} \subset \mathbb{S}^{1}$ is the set of $n$ equally spaced points ( $n$ is even). The lower bound then follows by Theorem 1.2. In fact, one can show that $c_{j}(2 k) \geq 2^{-j}$ : to obtain this inequality consider the polytope $\mathcal{B}_{2 k}(Z)$ where $Z=Y \cup(Y+\pi)$ and $Y$ lies in an arc of length at most $\phi_{k}$ as defined in Theorem 1.2.

It was observed by G. Ziegler [24] that the bound $c_{j}(d) \geq 2^{-j}$ can also be obtained by considering the family of cs polytopes $P_{n}=\operatorname{conv}\left(Q_{n} \cup\left(-Q_{n}\right)\right)$, where $Q_{n}$ is the $d$-dimensional cyclic polytope whose vertices are $\left(i, i^{2}, \ldots, i^{d}\right)$ for $1 \leq i \leq n$.

\section{Concluding Remarks}

We close the paper with three additional remarks on the face numbers of centrally symmetric polytopes and several open questions.

\subsection{The Upper Half of the Face Vector}

Theorems 1.3 and 1.4 provide estimates on $\operatorname{fmax}(2 k, n ; j)$-the maximal possible number of $j$-faces that a cs $2 k$-polytope on $n$ vertices can have-for $j \leq k-1$. What can be said about $\operatorname{fmax}(2 k, n ; j)$ for $j \geq k$ ? Here we prove that for every $k \leq j<2 k$, the value of $\operatorname{fmax}(2 k, n ; j)$ is of the order of $n^{k}$.

Theorem 7.1 For every positive even integer $d=2 k$ and an integer $k \leq j<2 k$, there exist positive constants $\gamma_{j}(d)$ and $\Gamma_{j}(d)$ such that

$$
\gamma_{j}(d)+o(1) \leq \frac{\operatorname{fmax}(d, n ; j)}{\left(\begin{array}{l}
n \\
k
\end{array}\right)} \leq \Gamma_{j}(d)+o(1) \quad \text { as } n \rightarrow+\infty .
$$


Proof The upper bound estimate follows from the Upper Bound Theorem [16] which holds for all polytopes. To verify the lower bound, consider a cs $2 k$-polytope $P_{n}$ on $n$ vertices that satisfies $f_{k-1}\left(P_{n}\right)=\operatorname{fmax}(2 k, n ; k-1)$. As in the proof of Proposition 2.2 we can assume that $P_{n}$ is a simplicial polytope. Let

$$
h\left(P_{n}\right)=\left(h_{0}\left(P_{n}\right), h_{1}\left(P_{n}\right), \ldots, h_{2 k}\left(P_{n}\right)\right)
$$

be the $h$-vector of $P_{n}$ (see for instance [23, Chap. 8]), that is, the vector whose entries are defined by the polynomial identity

$$
\sum_{i=0}^{d} h_{i}\left(P_{n}\right) x^{2 k-i}=\sum_{i=0}^{d} f_{i-1}\left(P_{n}\right)(x-1)^{2 k-i} .
$$

Equivalently,

$$
f_{j-1}\left(P_{n}\right)=\sum_{i=0}^{j}\left(\begin{array}{c}
2 k-i \\
2 k-j
\end{array}\right) h_{i}\left(P_{n}\right), \quad j=0,1, \ldots, 2 k .
$$

The $h$-numbers of a simplicial polytope are well-known to be nonnegative and symmetric [23, Chap. 8], that is, $h_{j}\left(P_{n}\right)=h_{2 k-j}\left(P_{n}\right)$ for $j=0,1, \ldots, 2 k$. Moreover, McMullen's proof of the UBT implies that the $h$-numbers of any simplicial $2 k$ polytope with $n$ vertices satisfy

$$
h_{j} \leq\left(\begin{array}{c}
n-2 k+j-1 \\
j
\end{array}\right)=O\left(n^{j}\right), \quad \text { for } 0 \leq j \leq k .
$$

Substituting these inequalities into (11) for $j=k-1$ and using that

$$
f_{k-1}\left(P_{n}\right)=\operatorname{fmax}(2 k, n ; k-1)=\Omega\left(n^{k}\right)
$$

by Theorem 1.4, we obtain

$$
h_{k}\left(P_{n}\right)=\Omega\left(n^{k}\right) .
$$

Together with nonnegativity of $h$-numbers and (11), this implies that

$$
\operatorname{fmax}(2 k, n ; j) \geq f_{j}\left(P_{n}\right)=\Omega\left(n^{k}\right) \quad \text { for all } k \leq j<2 k,
$$

as required.

\section{2-Faces of $\mathcal{B}_{6}$}

We provide some additional estimates on the extent to which $\mathcal{B}_{6}$ is 3-neighborly.

Theorem 7.2 Let $t_{1}, t_{2}, t_{3} \in \mathbb{R}$ be such that the points $z_{1}=e^{i t_{1}}, z_{2}=e^{i t_{2}}$, and $z_{3}=$ $e^{i t_{3}}$ are distinct and lie on an arc of the unit circle of length at most $\arccos (1 / 8)$. Then the convex hull of the set $\left\{\mathrm{SM}_{6}\left(t_{1}\right), \mathrm{SM}_{6}\left(t_{2}\right), \mathrm{SM}_{6}\left(t_{3}\right)\right\}$ is a 2-dimensional face of $\mathcal{B}_{6}$. 
Proof As in Proposition 6.1 and Theorem 1.2, the proof reduces to verifying the following statement:

Let $z_{1}, \ldots, z_{6} \in \mathbb{C}$ be distinct points that lie on an arc of the unit circle $|z|=1$ of length at most $\arccos (1 / 8)$. Let $D(z)$ be a raked self-inversive polynomial of degree 10 such that $D\left(z_{j}\right)=0$ for $j=1, \ldots, 6$. Then none of the remaining roots of $D$ has the absolute value of 1 .

Let $z_{7}, z_{8}, z_{9}$, and $z_{10}$ be the remaining roots of $D$ (some of the roots may coincide). Let $\Phi$ be an arc of the unit circle $|z|=1$ of length $l \leq \arccos (1 / 8)$ that contains $z_{1}, \ldots, z_{6}$. Consider the line $L$ through the origin that bisects $\Phi$. Since $D$ is a raked polynomial, we must have

$$
\sum_{j=1}^{10} z_{j}=\sum_{j=1}^{10} z_{j}^{3}=0
$$

cf. Lemma 3.3. Let $\Sigma_{1}$ be the sum of the orthogonal projections of $z_{1}, \ldots, z_{6}$ onto $L$ and let $\Sigma_{2}$ be the sum of the orthogonal projections of $z_{7}, \ldots, z_{10}$ onto $L$, so

$$
\Sigma_{1}+\Sigma_{2}=0
$$

As $\cos l \geq 1 / 8$, we have $\cos (l / 2) \geq 3 / 4$, and hence

$$
\left|\Sigma_{2}\right|=\left|\Sigma_{1}\right| \geq 6 \cdot \frac{3}{4}=\frac{9}{2} .
$$

Therefore, for at least one of the roots of $D$, say, $z_{9}$ we have $\left|z_{9}\right|>1$. Then, for another root of $D$, say, $z_{10}$ we have $\left|z_{10}\right|=1 /\left|z_{9}\right|<1$, cf. Lemma 3.3. If $\left|z_{7}\right|>1$ then $\left|z_{8}\right|<1$ and we are done. Hence the only remaining case to consider is $\left|z_{7}\right|=$ $\left|z_{8}\right|=1$. In this case, by (13), we should have $\left|z_{9}\right| \geq 2$. Using that $z_{10}=1 / \overline{z_{9}}$ we obtain

$$
\left|z_{9}^{3}+z_{10}^{3}\right|=\left|z_{9}^{3}\right|+\left|z_{10}^{3}\right|>8=\sum_{j=1}^{8}\left|z_{j}\right|^{3} \geq\left|\sum_{j=1}^{8} z_{j}^{3}\right|,
$$

which contradicts (12).

\subsection{Lower Bounds for $c_{j}(d)$}

I. Bárány [1] suggested to the authors to look at the following family of polytopes as a source of cs polytopes with many faces. Consider $\mathbb{R}^{2 k}$ as a direct sum of $k$ copies of $\mathbb{R}^{2}$ :

$$
\mathbb{R}^{2 k}=\mathbb{R}^{2} \oplus \cdots \oplus \mathbb{R}^{2}
$$

and let $C_{i}$ denote the unit circle $\mathbb{S}^{1} \subset \mathbb{R}^{2}$ in the $i$ th copy. Let $n=k m$ be the multiple of an even integer $m \geq 4$ and let $X_{i} \subset C_{i}$ be a set of $m$ equally spaced points. Define

$$
P_{n, k}:=\operatorname{conv}\left(\bigcup_{i=1}^{k} X_{i}\right) \text {. }
$$


In other words, $P_{n, k}$ is the join of $k$ cs $m$-gons. Thus $P_{n, k}$ is a cs $2 k$-dimensional polytope with the property that for every subset of indices $I \subset\{1, \ldots, k\}$ and a choice of points $x_{i} \in X_{i}$, one for each $i \in I$, the set $\operatorname{conv}\left(x_{i}: i \in I\right)$ is a face of $P_{n, k}$. Hence

$$
f_{j}\left(P_{n, k}\right) \geq\left(\begin{array}{c}
k \\
j+1
\end{array}\right)\left(\frac{n}{k}\right)^{j+1} \quad \text { for } 0 \leq j \leq k-1,
$$

which gives the bound

$$
c_{j}(2 k) \geq \frac{k(k-1) \cdots(k-j)}{k^{j+1}} .
$$

We note that for $j=1$ the obtained bound $c_{1}(2 k) \geq 1-k^{-1}$ is weaker than the bound $c_{1}(2 k) \geq 1-(2 k-1)^{-1}$ of Theorem 1.3. Also for $j=k-1$, the obtained bound $c_{k-1}(2 k)=k ! / k^{k} \approx e^{-k}$ is weaker than the bound $c_{k-1}(2 k) \geq 2^{-k}$ following from the proof of Theorem 1.4. Still, we can conclude that for any fixed $j$,

$$
\lim _{d \rightarrow+\infty} c_{j}(2 k)=1
$$

\subsection{Open Questions}

There are several natural questions that we have not been able to answer so far.

- It seems plausible that $\psi_{k}$ in Theorem 1.1 satisfies

$$
\psi_{k}=\frac{2 k-2}{2 k-1} \pi
$$

but we are unable to prove that.

- We do not know what is the best value of $\phi_{k}$ in Theorem 1.2 for $k>2$ nor the values of $c_{j}(d)$ in Theorem 1.4.

- The most intriguing question is, of course, whether the class of polytopes $\mathcal{B}_{2 k}(X)$ indeed provides (asymptotically or even exactly) polytopes with the largest number of faces among all centrally symmetric polytopes with a given number of vertices.

Acknowledgements The authors are grateful to J.E. Goodman, R. Pollack, and J. Pach, the organizers of the AMS-IMS-SIAM Summer Research Conference "Discrete and Computational Geometry-twenty years later" (Snowbird, June 2006), where this project started, to L. Billera for encouragement, to I. Bárány for suggesting the example of section 7.3, and to the anonymous referee for helpful comments.

\section{References}

1. Bárány, I.: personal communication (2007)

2. Barvinok, A.: A Course in Convexity. Graduate Studies in Mathematics, vol. 54. American Mathematical Society, Providence (2002)

3. Björner, A.: personal communication (2006)

4. Billera, L.J., Lee, C.W.: A proof of the sufficiency of McMullen's conditions for $f$-vectors of simplicial convex polytopes. J. Comb. Theory Ser. A 31, 237-255 (1981)

5. Caratheodory, C.: Über den Variabilitatsbereich det Fourierschen Konstanten von Positiven harmonischen Furktionen. Rend. Circ. Mat. Palermo 32, 193-217 (1911) 
6. Danzer, L., Grünbaum, B.: Über zwei Probleme bezüglich konvexer Körper von P. Erdös und von V.L. Klee. Math. Z. 79, 95-99 (1962) (in German)

7. Donoho, D.L.: High-dimensional centrosymmetric polytopes with neighborliness proportional to dimension. Discrete Comput. Geom. 35, 617-652 (2006)

8. Donoho, D.L.: Neighborly polytopes and sparse solutions of underdetermined linear equations. Preprint (2004)

9. Donoho, D.L., Tanner, J.: Counting faces of randomly-projected polytopes when the projection radically lowers dimension. Preprint, math.MG/0607364

10. Gale, D.: Neighborly and cyclic polytopes. In: Proc. Sympos. Pure Math., vol. VII, pp. $225-232$. American Mathematical Society, Providence (1963)

11. Grünbaum, B.: Convex polytopes, 2nd edn. (prepared and with a preface by V. Kaibel, V. Klee and G.M. Ziegler). Graduate Texts in Mathematics, vol. 221. Springer, New York (2003)

12. Grünbaum, B., Motzkin, T.S.: On polyhedral gaps. In: Proc. Sympos. Pure Math., vol. VII, pp. 285290. American Mathematical Society, Providence (1963)

13. Katona, G.O.H.: A theorem of finite sets. In: Theory of Graphs, Proc. Colloq., Tihany, 1966, pp. 187207. Academic Press, New York (1968)

14. Kruskal, J.B.: The number of simplices in a complex. In: Mathematical Optimization Techniques, pp. 251-278. University of California Press, Berkeley (1963)

15. Linial, N., Novik, I.: How neighborly can a centrally symmetric polytope be? Discrete Comput. Geom. 36, 273-281 (2006)

16. McMullen, P.: The maximum numbers of faces of a convex polytope. Mathematika 17, 179-184 (1970)

17. Motzkin, T.S.: Comonotone curves and polyhedra. Bull. Am. Math. Soc. 63, 35 (1957)

18. Rudelson, M., Vershynin, R.: Geometric approach to error correcting codes and reconstruction of signals. Int. Math. Res. Not. 64, 4019-4041 (2005)

19. Sheil-Small, T.: Complex Polynomials. Cambridge Studies in Advanced Mathematics, vol. 75. Cambridge University Press, Cambridge (2002)

20. Smilansky, Z.: Convex hulls of generalized moment curves. Isr. J. Math. 52, 115-128 (1985)

21. Smilansky, Z.: Bi-cyclic 4-polytopes. Isr. J. Math. 70, 82-92 (1990)

22. Stanley, R.: The number of faces of simplicial convex polytopes. Adv. Math. 35, 236-238 (1980)

23. Ziegler, G.M.: Lectures on Polytopes. Graduate Texts in Mathematics, vol. 152. Springer, New York (1995)

24. Ziegler, G.M.: personal communication (2007) 